\title{
The Self-Administered Version of the Structured INTERVIEW fOR DisORDers Of Extreme Stress (SIDES-SR) in A CLINICAL AND NON-CLINICAL PORTUgUeSe SAMPLE
}

Rute Pires ${ }^{1,2}$, Ana Sousa Ferreira ${ }^{1,3}$, Joana Henriques-Calado ${ }^{1,2}$, Bruno Gonçalves ${ }^{1,2}, \&$ Marco Paulino ${ }^{1,4}$

${ }^{1}$ Faculdade de Psicologia, Universidade de Lisboa, Alameda da Universidade, 1649-013 Lisboa, Portugal; ${ }^{2}$ CICPSI, Faculdade de Psicologia, Universidade de Lisboa, Alameda da Universidade, 1649-013 Lisboa, Portugal; ${ }^{3}$ Instituto Universitário de Lisboa - Business Research Unit (BRU-IUL), Portugal; ${ }^{4}$ Faculdade de Medicina, Universidade de Lisboa, Avenida Professor Egas Moniz, 1649-028 Lisboa, Portugal

rpires@psicologia.ulisboa.pt asferreira@psicologia.ulisboa.pt joanahenriquescalado@gmail.com bgoncalves@psicologia.ulisboa.pt mpaulino@netcabo.pt

\section{INTRODUCTION}

DESNOS (Disorders of extreme stress not otherwise specified; Pelcovitz et al., 1997): constellation of symptoms and altered beliefs that emerge as a consequence of prolonged, repeated adverse interpersonal experiences (e.g., child abuse, domestic violence).

Traumatic interpersonal experiences compromise the individual's self-development, particularly when occur during developmentally vulnerable periods (i.e., childhood or adolescence), and when they are inflicted by caregivers or in the context of other intimate relationships (Cook et al., 2017; Ford, Grasso, Greene, Levine, \& Spinazzola, 2013; Luxenberg, Spinazzola, van der Kolk, 2001).

The DSM-5 (APA, 2013) included DESNOS psychopathology outcomes as specific features of PTSD, however, findings from clinical and research studies support that a diagnosis of PTSD fails to adequately capture DESNOS' symptoms (Luxenberg et al., 2001; Pelcovitz et al., 1997; van der Kolk, 2014).

Pelcovitz et al. (1997) developed the Structured Interview for Disorders of Extreme Stress (SIDES), a rationally derived structured interview that assesses DESNOS symptomatology. In its prevailing form, there are two versions of the instrument, the interview (SIDES-R) and the self-administered version (SIDES-SR; Spinazzola, Blaustein, Kisiel, \& van der Kolk, as cited in Luxenberg, 2001).

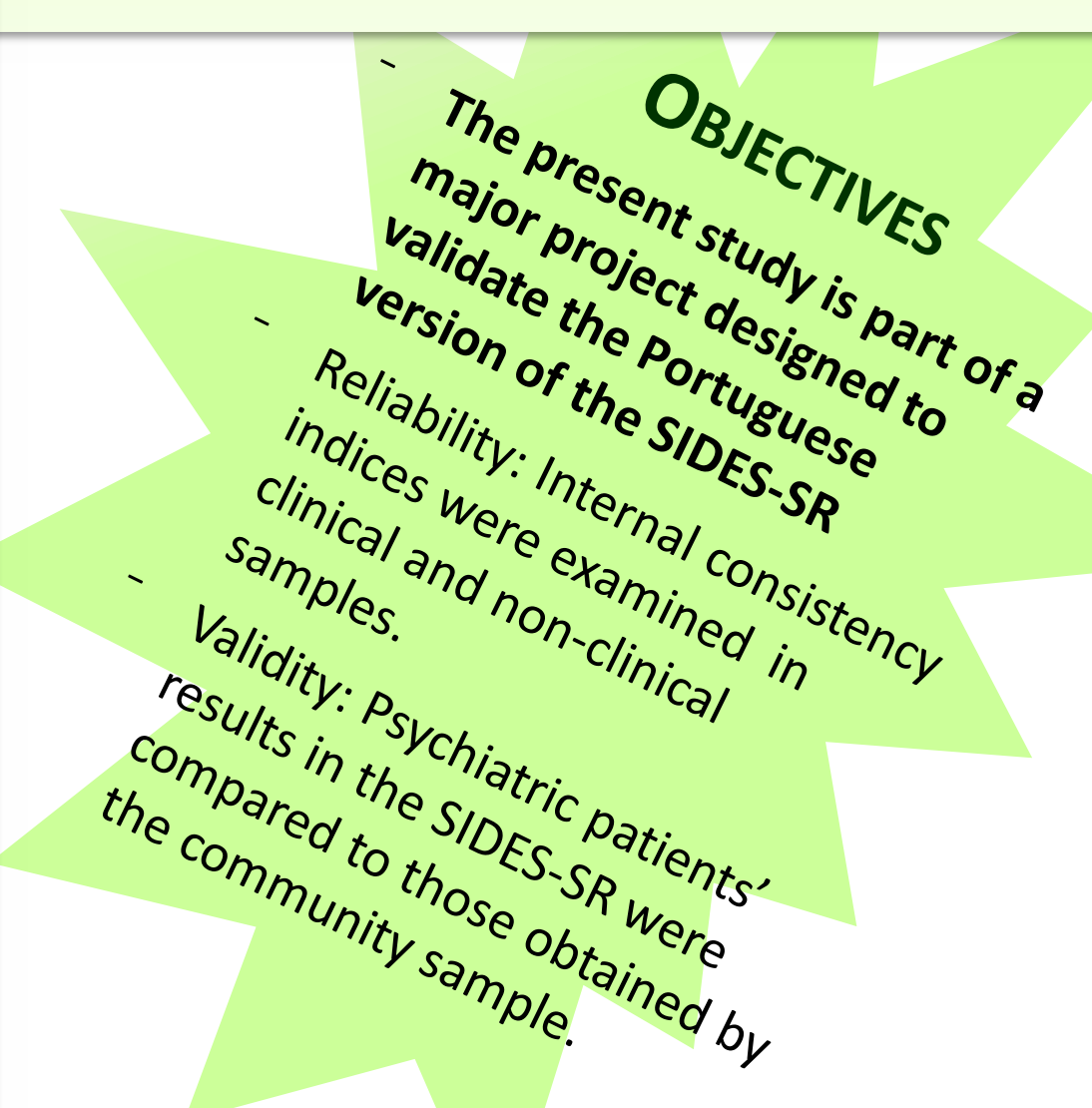

\section{METHOD}

PARTICIPANTS: Community sample: $N=814 ; M_{\text {age }}=40.09, S D=14.25,39.2 \%$ male, $60.8 \%$ female; Clinical sample: $N=310 ; M_{\text {age }}=42.49, S D=12.47,57.7 \%$ male, 42.3\% female). PROCEDURES: The community and clinical samples' data collection was approved by the Ethic Committee of the Faculty of Psychology and, in the case of the clinical sample, by the host institutions involved. The participation in the study was informed, consented, anonymous and voluntary. MEASURES: The SIDES-SR is a 45 item self-report measure which assesses lifetime and current presence of DESNOS, in addition to current symptom severity (past month). Lifetime presence of DESNOS is rated on a dichotomic scale (Yes/No) and current presence in the past month and symptom severity are rated on a 4-point Likert scale ranging from 0 (none; not at all) to 3 (maximum severity). Scores of 2 or above suggest clinical severity of the symptoms presented.

The SIDES-SR characterizes the DESNOS' six symptomatology clusters: I) ALTERATIONS IN REGULATION OF AFFECT AND IMPULSES, II) ALTERATIONS IN ATTENTION OR consciousness, III) Alterations in Self-perception, IV) Alterations in relationships With others, V) Somatization, Vi) Alterations in SYStems of MEaning.

\section{RESULTS}

Table 1. SIDES-SR scales' means $(M)$, standard deviations $(S D)$, Cronbach's alphas $(\alpha)$ in the community and clinical samples

\begin{tabular}{|c|c|c|c|c|c|c|c|}
\hline & $\begin{array}{c}\text { SIDES-R } \\
\text { (Pelcovitz et al., } \\
\text { 1997) }\end{array}$ & \multicolumn{3}{c|}{$\begin{array}{c}\text { SIDES-SR Portuguese version } \\
\text { Community } \\
(N=814)\end{array}$} & \multicolumn{3}{c|}{$\begin{array}{c}\text { Clinical } \\
(N=310)\end{array}$} \\
\hline & $\boldsymbol{\alpha}$ & $\mathbf{M}$ & SD & $\boldsymbol{\alpha}$ & $\mathbf{M}$ & SD & $\boldsymbol{\alpha}$ \\
\hline I & .90 & .18 & .33 & .90 & .67 & .61 & .77 \\
\hline II & .76 & .18 & .50 & .68 & .67 & .82 & .62 \\
\hline III & .77 & .07 & .21 & .78 & .37 & .52 & .69 \\
\hline IV & .77 & .15 & .37 & .67 & .48 & .66 & .62 \\
\hline V & .88 & .06 & .21 & .69 & .26 & .51 & .71 \\
\hline VI & .78 & .16 & .45 & .69 & .53 & .86 & .67 \\
\hline
\end{tabular}

\section{DISCUSSION}

- Internal consistency indices above .60 , lower than the original ones, but similar to other studies (.68-.82, Luxenberg et al., 2001; .67-.71, Scoboria et al., 2008).

- The SIDES-SR results highly differentiated a community and a clinical sample, with the clinical sample reporting, as expected, more severe symptomatology.

- Moderate effect sizes suggest that the SIDES-SR adequately captures the severity of DESNOS symptomatology.

- Results support SIDES-SR relevance for clinical practice in the assessment of the DESNOS diagnosis.

\begin{tabular}{|c|c|c|c|c|c|c|}
\hline \multirow{13}{*}{ 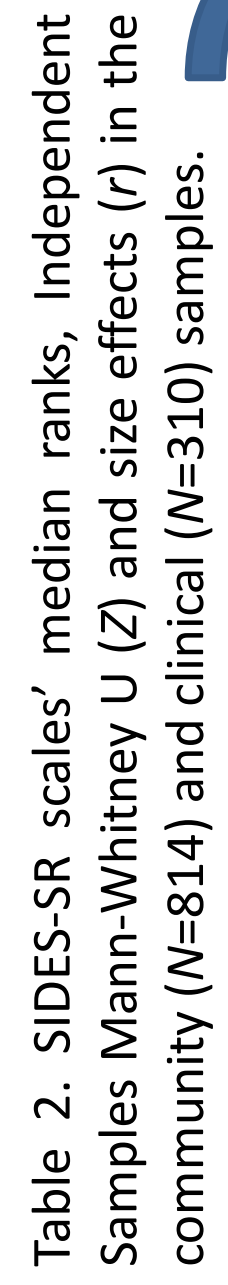 } & & & Mean Ranks & $Z$ & $p$ & $r$ \\
\hline & \multirow{2}{*}{ I } & Community & 450.49 & \multirow{2}{*}{-14.05} & \multirow{2}{*}{.000} & \multirow{2}{*}{.44} \\
\hline & & Clinical & 717.96 & & & \\
\hline & \multirow{2}{*}{ II } & Community & 489.87 & \multirow{2}{*}{-11.78} & \multirow{2}{*}{.000} & \multirow{2}{*}{.36} \\
\hline & & Clinical & 674.36 & & & \\
\hline & \multirow{2}{*}{ III } & Community & 483.32 & \multirow{2}{*}{-12.25} & \multirow{2}{*}{.000} & \multirow{2}{*}{.38} \\
\hline & & Clinical & 674.47 & & & \\
\hline & \multirow{2}{*}{ IV } & Community & 500.14 & \multirow{2}{*}{-9.70} & \multirow{2}{*}{.000} & \multirow{2}{*}{.30} \\
\hline & & Clinical & 656.71 & & & \\
\hline & \multirow{2}{*}{ V } & Community & 504.86 & \multirow{2}{*}{-7.78} & \multirow{2}{*}{.000} & \multirow{2}{*}{.24} \\
\hline & & Clinical & 608.40 & & & \\
\hline & \multirow{2}{*}{ VI } & Community & 506.57 & \multirow{2}{*}{-7.88} & \multirow{2}{*}{.000} & \multirow{2}{*}{.24} \\
\hline & & Clinical & 618.37 & & & \\
\hline
\end{tabular}

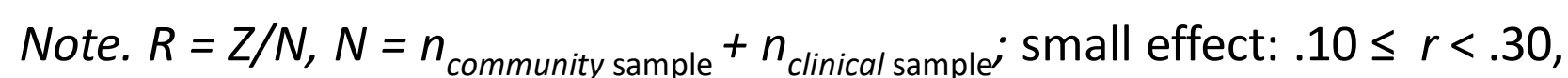
medium effect: $.30 \leq r<.50$, large effect: $r \geq .50$

- Study limitations: the composition of the clinical sample, in which the diagnosis of substance use is overrepresented.

Future directions: balance diagnoses; expand the validity studies (e.g., factor structure, history of interpersonal trauma, associations with PTSD measures)

\section{REFERENCES}

American Psychiatric Association (2013). Diagnostic and statistical manual of mental disorders (5 $5^{\text {th }}$ ed.). Arlington, VA: American Psychiatric Publishing.

Cook, A., Spinazzola, J., Ford, J., Lanktree, C., Blaustein, M., Cloitre, M.,... \& Mallah, K. (2017). Complex trauma in children and adolescents. Psychiatric annals, 35(5), 390-398. doi: 10.3928/00485713-20050501-05

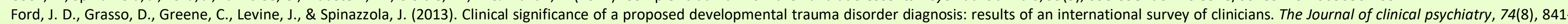
849. doi: $10.4088 / 1 C P 12 m 08030$.

Luxenberg, T., Spinazzola, J., \& Van der Kolk, B. A. (2001). Complex trauma and disorders of extreme stress (DESNOS) diagnosis, part one: Assessment. Directions in psychiatry, 21(25), 373-392.

Pelcovitz, D., Van der Kolk, B., Roth, S., Mandel, F., Kaplan, S., \& Resick, P. (1997). Development of a criteria set and a structured interview for disorders of extreme stress (SIDES). Journal of traumatic stress, 10(1), 3-16 Scoboria, A., Ford, J., Lin, H. J., Frisman, L. (2008). Exploratory and confirmatory factor analyses of the structured interview for disorders of extreme stress. Assessment, 15(4), 404-25. doi: 10.1177/1073191108319005 van der Kolk, B. (2014). The body keeps the score: Mind, brain and body in the transformation of trauma. Penguin UK. 